\title{
STOC: Energy Cost Models of Smartphones for Task Offloading to the Cloud
}

\author{
Rahitha $K^{1}$, Gireesh $\mathbf{T} \mathbf{K}^{2}$ \\ ${ }^{1,2}$ AWH Engineering College, Calicut University, Department of Computer Science \& Engineering Kuttikkatoor, Kozhikode, India
}

\begin{abstract}
Improving computing ability of smartphones and also prolonging their battery life can be achieved by offloading the task to the cloud. However, task offloading introduces a communication cost for those devices. Therefore, consideration of the communication cost is crucial for the effectiveness of task offloading. To make task offloading beneficial, one of the challenges is to estimate the energy consumed in communication activities of task offloading. Accurate energy estimation models will enable these devices to make the right decisions as to whether or not to perform task offloading, based on the energy cost of the communication activities. If the offloading process consumes less energy than processing the task on the device itself, then the task is offloaded to the cloud Here energy models during task offloading can be constructed during both uploading and downloading process using 3G/4G and Wifi. Also an analysis report is prepared giving detailed information of the energy consumed during uploading and downloading video files. Storage as a service (SaaS) aspect of the cloud is utilized for task offloading.
\end{abstract}

Keywords: Mobile Computing, Cloud Computing, Smartphones, Offloading Decision, Energy Saving, WLAN Energy, 3G Energy, 4G Energy, Energy Estimation

\section{Introduction}

The main constraints for smartphones are limited battery energy, low processing capability, and limited memory capacity. Since many years, the limited battery energy constraint has not been satisfactorily addressed. With the emergence of high-speed broadband wireless Internet access, task offloading has become a promising technique to reduce energy consumption in smartphone. That is because highspeed networks increase the connection availability to the computing resources behind the Internet.

Using the offloading technique, smartphones can offload their heavy tasks to remote machines and save their energy of executing the task locally. In the era of Cloud Computing (CC), the energy constraint on smartphones can be eased off by offloading heavy tasks from smartphones to the cloud. Over the last few years, rapid progresses in semiconductor technologies have alleviated some of those constraints. However, the limited battery energy constraint has not been satisfactorily addressed. According to Moore's law, the number of transistors on an integrated circuit doubles every two years. In contrast, battery capacity increases only by 5 percent every year. This fact implies that the gap between energy demand and supply grows by 4percent annually.

In the recent years, the problem has become prevalent among smartphone users, while the smartphones are becoming increasingly popular because of their capabilities and functionalities. With powerful operating systems (e.g., Windows Mobile, Android, Apple iOS, BlackBerry, and

Symbian), smartphones are able to run advance applications that are almost similar to desktop computer applications. Each smartphone application performs a series of tasks, with each task executing specific computations on a given data. The need to reduce the energy consumption of smartphones has been attracting efforts from many researchers.
Many methodologies and techniques have been proposed in literature. Smart batteries, power scheduling, efficient operating systems and applications, efficient graphical user interfaces, energy-aware communication protocols, and task offloading are all examples of these methodologies and technique. The mobile device can save energy by offloading heavy tasks to the cloud, and then the cloud executes the tasks and provides the mobile device with the results. For example, a smartphone can upload a video file to a cloud and request to encode the file into a desired format fitting the smartphone capability with less energy consumption than doing the encoding on the device.

The rest of the paper is organized as follows. Section 2 presents the literature survey, followed by Task offloading to the cloud i.e. video encoding. The system architecture and algorithm are included in section 3.1 and 3.2 followed by conclusion and future work of this paper

\section{Literature Survey}

Literature survey deals with the related techniques which helps in reducing power consumption and increasing the processing efficiency of smartphones. Power management is an important aspect of mobile computing. Instead of hardware approach M. Othman[1] propose a different approach of power conservation strategy for mobile computers which is based on the concept of load sharing. User jobs are transferred from a mobile host to a fixed host to reduce power consumption by the CPU. Simulation results show that under suitable conditions, transferring job can extend battery lifetime by up to $20 \%$. Transferring jobs to a fixed host does not only extend battery lifetime but also gives users access to faster machines, hence improving job response time.

The offloading technique can be categorized into three major approaches based on the type of the remote machine. The first approach is the offloading to a web proxy [2] where a 


\section{International Journal of Science and Research (IJSR) \\ ISSN (Online): 2319-7064}

Index Copernicus Value (2013): 6.14 | Impact Factor (2015): 6.391

proxy works as an intermediary machine between a web server and a mobile device. The mobile device sends a web request to the proxy and the proxy delivers the content to the mobile device after performing the desired modification to the content, such as multimedia coding. BitTorrent-based file-sharing is already available for mobile phones; however, its energy profile makes it difficult to use it for transferring large amount of data. This paper analyzes an alternative cloud-based solution that uses a remote server to download content via BitTorrent and transfer it to the mobile device in a transparent and energy efficient way.

Kun Yang and Shumao $\mathrm{Ou}[3]$ proposes a novel offloading service that can seamlessly offload some of the tasks of a mobile application from an $\mathrm{MH}$ to nearby, resource-rich PCs (called surrogates). The system architecture and key components of the proposed offloading service are presented, prototyped, and evaluated. The results of experiments and simulations have demonstrated the effectiveness and efficiency of this offloading service for mobile applications. The offloading approach considers a combination of multiple resources including CPU, memory, and communication cost (i.e., bandwidth resource); and aims to reserve these precious $\mathrm{MH}$ resources as much as possible. It provides mobility support and enables multiple surrogates to be utilized. The objective is to the reduce response time for a user application running on a resource-constrained $\mathrm{MH}$.

In this paper, Jayant Baliga, Robert W. A. Ayre , Kerry Hinton [4] present an analysis of energy consumption in cloud computing. The analysis considers both public and private clouds, and includes energy consumption in switching and transmission as well as data processing and data storage. They show that energy consumption in transport and switching can be a significant percentage of total energy consumption in cloud computing. Cloud computing can enable more energy-efficient use of computing power, especially when the computing tasks are of low intensity or infrequent.

Shivani Hiwarekar [5] discusses that with the rapid development of new and innovative applications for mobile devices like smartphones, advances in battery technology have not kept pace with rapidly growing energy demands. Thus energy consumption has become a more and more important issue of mobile devices. To meet the requirements of saving energy, it is critical to monitor and analyze the energy consumption of applications on smartphones. For this purpose, they developed a smart energy monitoring system called SEMO for smartphones using Android operating system. It can profile mobile applications with battery usage information, which is vital for both developers and users.

Miettinen et al. [6] model the energy cost at the system level when a smartphone performs communication and computation. The offloading decision is made by comparing the energy cost of mobile communication and computation for a given task. This work shows the impact of communication bandwidth on task offloading, and illustrates that offloading is beneficial if the task has heavy computation and needs low communication. However, the energy cost in this work lacks experimental validation, and the impacts of Internet protocols and network interfaces on the energy cost have not been considered. This paper discusses the energy efficiency of mobile clients in cloud computing. Cloud computing is a promising technology which can offer many benefits for mobile devices. The main focus is on computation offloading, which can be used to save energy for the battery powered devices.

Huang et al. [7] showed the distinct levels of power consumption by tracing the radio resources and power consumptions of the smartphones for $3 \mathrm{G}$ and $4 \mathrm{G}$ networks, respectively. X.Zhang et.al[8] proposes a new elastic application model that enables seamless and transparent use of cloud resources to augment the capability of resourceconstrained mobile devices. The salient features of this model include the partition of a single application into multiple components called weblets, and a dynamic adaptation of weblet execution configuration. While a weblet can be platform independent (e.g., Java or .Net bytecode or Python script) or platform dependent (native code), its execution location is transparent it can be run on a mobile device or migrated to the cloud, i.e., run on one or more nodes offered by an IaaS provider. Thus, an elastic application can augment the capabilities of a mobile device including computation power, storage, and network bandwidth, with the light of dynamic execution configuration according to devices status including CPU load, memory, battery level, network connection quality, and user preferences. This paper presents the motivation behind developing elastic applications and their architecture including typical elasticity patterns and cost models that are applied to determine the elasticity patterns.

\section{Task offloading to the Cloud}

Here we deal with the techniques for reducing the power consumption and increasing the processing capability and memory capacity of smartphones by offloading the task to the real cloud. It also discusses energy models generated in the form of graphical representation during the uploading and downloading processes. Both $3 \mathrm{G} / 4 \mathrm{G}$ and Wi-Fi network interfaces are efficiently utilized in accordance with user's preference. The energy consumed during the network activities of task offloading is accurately recorded as analysis report. The details of power both uploaded and downloaded video files are elaborately recorded.

The models can be used to estimate the energy consumed in a smartphone to perform task offloading in file downloading using WLAN and 3G/4G network interfaces; and file uploading using WLAN and $3 \mathrm{G} / 4 \mathrm{G}$ network interfaces.

\subsection{System Architecture}

This section shows the system architecture of the system. 


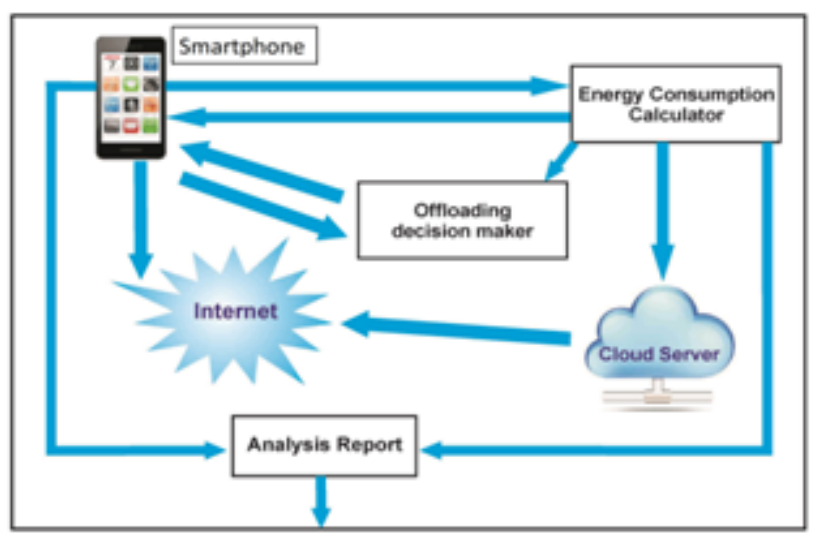

The system consists of two major parts, smartphones (i.e., user equipment, UE) and Cloud Computing (CC), both linked to the Internet. The smartphones are connected to the Internet through a WLAN access point or a cellular data network base station $(3 \mathrm{G} / 4 \mathrm{G})$. These smartphones provide all of mobile computing functionalities to the end users via different applications. On the other hand, the $\mathrm{CC}$ part consists of cloud data center and cloud provider, which are accessible through the Internet. The cloud provides the end users (e.g., smartphone users) with all of the CC functionalities that are needed for mobile computing. In the offloading technique, smartphones access the cloud via the Internet. Therefore, offloading is considered as a Network Related Application (NRA).Both the network interfaces (i.e., $3 \mathrm{G} / 4 \mathrm{G}$ and WLAN) are considered because each of these interfaces has its own characteristics, such as supported data rate. As a result, each network interface consumes unequal amount of energy.

The mobile device can save energy by offloading heavy tasks to the cloud, and then the cloud executes the tasks and provides the mobile device with the results. Here it is being performed as a smartphone can upload a video file to a cloud and request to encode the file into a desired format fitting the smartphone capability with less energy consumption than doing the encoding on the device. It is an impossible task for doing it in the mobile device itself. So the task is being performed by STOC.

The energy consumed when uploading the video file to the cloud and also while downloading the file are calculated. And also a graphical representation is generated which gives the energy consumption using both network interfaces $(3 \mathrm{G} / 4 \mathrm{G})$ \& Wi-Fi. The analysis report gives the detailed information about the uploaded and downloaded files and the energy consumption.

\subsection{Energy Cost Estimation}

Algorithm: To compute Energy cost

Input: File->input file

1. Start

2. Initialise before time to current mobile time. Call subbattery and save the output to Bbattety.

3. Calculate total transmitting bytes of mobile using net loss and assign this value to TotalTxBeforeTest.

4. Compute total receive bytes till now to TotalRxBeforeTest.
5. Send file I as a offloading task to third party.

6. After file uploaded follow step 7.

7. Set current time to Aftertime.

8. Set Time Diff=ATime-BTime.

9. Set Text Diff $=$ TotalTxAfterTest- TotalTxBeforeTest.

10. Set Txbps=(TextDiff $) /($ TimeDiff $/ 1000)$

11. Identify the connection type of mobile phone using connectivity manager.

12. Call subbattery and save the $o / p$ to Abattery.

13. Voltageloss $=$ Abattery - Bbattery.

14. Save the $\mathrm{o} / \mathrm{p}$ to $\mathrm{db}$ along with computed values and current $\mathrm{n} / \mathrm{w}$.

\section{$\underline{\text { Sub module setbattery ( ) }}$}

1. Start

2. Compute current voltage level of phone using battery manager service as save the value to level

3. Compute scaling factor and assign the value to scale

4. If level $=-1$ or scale $=-1$ then return so

5. Else compute $\mathrm{m}=($ level $/$ scale $) \mathrm{X} 100$

Return m

6. End

\section{Conclusion}

Task offloading to the cloud extends the capabilities of smartphone by overcoming various constraints such as limited battery energy, processing capability and memory capacity. But, in order to make the task offloading beneficial, the energy consumption during the network activities requires to be measured. Task offloading becomes beneficial only when the energy consumed in the offloading process is less than the energy consumed without it. Hence, the major challenge in task offloading is to estimate accurately the energy consumed during the network activities. In this work an android application was used for encoding video file to desired format along with estimation of energy consumption for network activities. Instead of estimating the energy consumption with the help of an embedded system, the battery status is framed directly from the mobile device and is plotted in the form of a graphical representation. Detailed report regarding name of file, size of file, power consumed during uploading or downloading and type of network used is also listed.

\section{Future Scope}

Smartphones are portable and handy, but all the applications that we handle on a desktop PC cannot be executed in a smartphone due to constraints like limited battery energy processing capability and memory capacity. STOC, a better alternative for a desktop PC, since a slight upgradation can solve as much as processes as a desktop PC. The energy and time consumption for various activities can also be forecasted which will change the history of smartphones in near future.

\section{References}

[1] M. Othman and S. Hailes, "Power Conservation Strategy for Mobile Computers Using Load Sharing," 
SIGMOBILE Mob. Comput.Commun. Rev., vol. 2, pp. 44-51, Jan. 1998.

[2] I. Kelenyi and J. K. Nurminen, "CloudTorrent - EnergyEfficientBitTorrent Content Sharing for Mobile Devices via Cloud Services,"in Proc. 7th IEEE Consumer Communications and NetworkingConf. (CCNC), 2010, pp. 1-2.

[3] K. Yang, S. Ou, and H.-H. Chen, "On Effective Offloading Services for Resource-Constrained Mobile Devices Running Heavier Mobile Internet Applications," IEEE CommunicationsMagazine, vol. 46, no. 1, pp. 5663, 2008.

[4] J. Baliga, R. W. A. Ayre, K. Hinton, and R. S. Tucker, "Green Cloud Computing: Balancing Energy in Processing, Storage, and Transport," Proceedings of the IEEE, vol. 99, no. 1, pp. 149-167, Jan. 2011.

[5] Fangwei Ding, Feng Xia, Wei Zhang, Xuhai Zhao," Monitoring Energy Consumption of Smartphones" Chengchuan Ma School of Software, Dalian University of Technology, Dalian 116620, China.

[6] A. P. Miettinen and J. K. Nurminen, "Energy Efficiency of Mobile Clients in Cloud Computing," in Proc. of the 2nd USENIX conference on Hot topics in cloud computing (HotCloud'10), 2010, p. 4.

[7] J. Huang, F. Qian, A. Gerber, Z. M. Mao, S. Sen, and O. Spatscheck,"A Close Examination of Performance and Power Characteristics of 4G LTE Networks," in Proceedings of the 10th International Conference on Mobile Systems, Applications, and Services, ser. MobiSys '12. ACM, 2012, pp. 225-238.

[8] X. Zhang, A. Kunjithapatham, S. Jeong, and S. Gibbs, "Towards an Elastic Application Model for Augmenting the Computing Capabilities of Mobile Devices with Cloud Computing," Mob. Netw. Appl., vol. 16, no. 3, pp. 270-284, Jun. 2011.

\section{Author Profile}

Rahitha K received the B Tech degrees in Information Technology from University of Calicut in 2011. She is currently persuading her $\mathrm{M}$ Tech in Computer Science and Engineering from University of Calicut.

Gireesh $\mathbf{T} \mathbf{K}$ received the B Tech and M Tech degrees in Computer Science and Engineering from university of Calicut and Visveswaraya Technological University - Belgaum in 2000 and 2008, respectively. During 2001- 2004, she was working as assistant professor in Amritha Institute of Computer Technology. He is working with AWH Engineering College since 2004. 